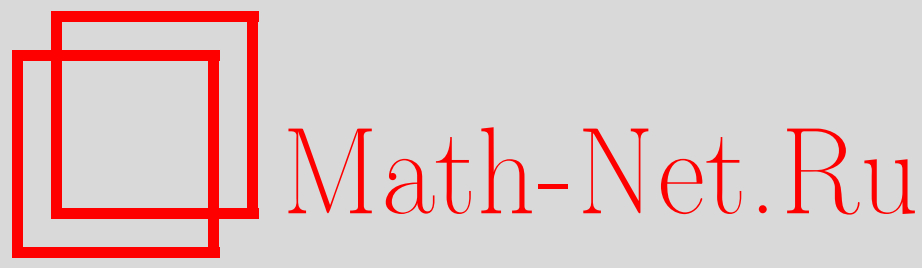

Н. А. Тюрин, Псевдоторические лагранжевы слоения торических и неторических многообразий Фано, ТМФ, 2010, том 162, номер 3, 307-333

DOI: https://doi.org/10.4213/tmf6473

Использование Общероссийского математического портала Math-Net.Ru подразумевает, что вы прочитали и согласны с пользовательским соглашением http://www.mathnet.ru/rus/agreement

Параметры загрузки:

IP: 3.81 .55 .215

26 апреля 2023 г., 05:30:18

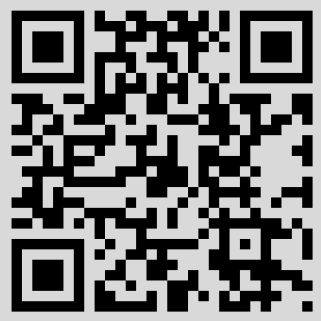




\title{
ФИЗИКА
}

Том 162, № 3

март, 2010

2010 г.

\author{
Н. А. Тюрин*
}

\section{ПСЕВДОТОРИЧЕСКИЕ ЛАГРАНЖЕВЫ СЛОЕНИЯ ТОРИЧЕСКИХ И НЕТОРИЧЕСКИХ МНОГООБРАЗИЙ ФАНО}

\begin{abstract}
Введено понятие псевдоторической структуры на симплектическом многообразии, обобщающее понятие торической структуры. Показано, что такие псевдоторические структуры могут существовать на торических и неторических симплектических многообразиях. Для первых это дает описание деформаций стандартных торических лагранжевых слоений, для вторых - существование лагранжевых слоений с особенностями, очень близких к торическим. Представлены примеры торических многообразий с разными псевдоторическими структурами, а также доказано, что некоторые неторические многообразия (гладкая комплексная квадрика) обладают такими структурами. В дальнейшем введение псевдоторических структур может оказаться полезным для обобщений методов геометрического квантования и зеркальной симметрии, хорошо работающих в торическом случае, на более широкий класс многообразий Фано.
\end{abstract}

Ключевые слова: торическое симплектическое многообразие, лагранжево слоение, невырожденная комплексная квадрика, тор Бора-Зоммерфельда.

\section{ВВЕДЕНИЕ}

В настоящей работе мы обсуждаем лагранжеву геометрию некоторых компактных симплектических многообразий, точнее, исследуем их лагранжевы слоения. Поэтому в большинстве случаев мы работаем с симплектическими торическими многообразиями, являющимися фазовыми пространствами соответствующих вполне интегрируемых систем (в работе [1] такие интегрируемые системы названы эффективными), и некоторыми подмногообразиями таких торических многообразий. Но торические многообразия не являются центральными в нашем обсуждении, они дают лишь подходящие рамки, в которых возможно предложить примеры и результаты. Ситуация же, изучаемая в настоящей работе, следующая: пусть симплектическое многообразие $(X, \omega)$ вещественной размерности $2 n$ допускает два разных по своей природе типа данных:

* Объединенный институт ядерных исследований, Дубна, Московская обл.; Московский государственный университет путей сообщения (МИИТ), Москва, Россия.

E-mail: ntyurin@theor.jinr.ru 
вещественная часть - набор вещественных морсовских функций $\left(f_{1}, \ldots, f_{k}\right)$ в инволюции;

комплексная часть - расслоение на симплектические подмногообразия вещественной размерности $2 k$ с базисным множеством $B$, индуцирующее отображение $\psi: X-B \rightarrow Y$ в торическое симплектическое многообразие $Y$ вещественной размерности $2(n-k)$ с гладкими в общем случае симплектическими слоями, удовлетворяющее некоторому дополнительному условию (см. определение в разделе 3 ; это условие является тривиальным в случае $k=n-1$, который рассматривается в разделах 1 и 2).

При этом вещественная и комплексная части согласованы в следующем смысле: для каждой $f_{i}$ ее гамильтоново векторное поле $X_{f_{i}}$ сохраняет каждый слой $\psi$, т.е. параллельно ему в каждой его точке.

Мы называем такой набор данных $\left(f_{1}, \ldots, f_{k}, \psi\right)$ псевдоторической структурой на симплектическом многообразии (см. определение). Сначала мы рассматриваем случай торических многообразий Фано, и ключевым моментом является то, что каждое торическое многообразие является также псевдоторическим. Это не тавтология, потому что каждое торическое многообразие Фано обладает разными псевдоторическими структурами, нас больше всего интересуют те, у которых $k=n-1$. Другими словами, мы имеем дело с отображением $\psi$ с симплектическими слоями, расслаивающим многообразие $M$ без симплектического подмножества (вещественной) коразмерности 4 (базисное множество пучка) над проективной прямой $\mathbb{C P}^{1}$, и с набором гладких морсовских функций $\left(f_{1}, \ldots, f_{n-1}\right)$, алгебраически независимых почти всюду и коммутирующих между собой, $\left\{f_{i}, f_{j}\right\}=0$. Условие согласованности означает, что для каждой функции $f_{i}$ гамильтоново векторное поле $X_{f_{i}}$ параллельно каждому дивизору $D_{p}=\overline{\psi^{-1}(p)}, p \in \mathbb{C P}^{1}$, являющемуся в общем случае гладким симплектическим дивизором в $(M, \omega)$.

В свете основного наблюдения из [2] представленная ситуация видится так: каждый гладкий дивизор $D_{p}$ вместе с ограничениями функций $\left(\left.f_{1}\right|_{D_{p}}, \ldots,\left.f_{n-1}\right|_{D_{p}}\right)$ доставляет вполне интегрируемую систему. В самом деле, коммутационные соотношения остаются верными после ограничения, откуда следует, что имеется полный набор интегралов для $\left(D_{p},\left.\omega\right|_{D_{p}}\right)$. Однако эти данные зависят от параметра $p \in \mathbb{C P}^{1}$, таким образом, мы имеем комплексное семейство интегрируемых систем. С другой стороны, ситуация уже не является классической, а похожа на систему для частицы со спином. Симплектическое многообразие $(X, \omega)$, снабженное набором данных $\left(f_{1}, \ldots, f_{n-1}, \psi\right)$, удовлетворяющих условию согласования, не является вполне интегрируемой системой, но может быть сведено к вполне интегрируемой системе с особенностями, причем на этом пути получаются разные системы. Как показано в работе [3], $n$-й специальный интеграл добавляется к набору $\left(f_{1}, \ldots, f_{n-1}\right)$, если гладкая вещественная функция $h \in C^{\infty}\left(\mathbb{C P}^{1}, \mathbb{R}\right)$ выбирается на параметризующей прямой, и значит, некоторое лагранжево слоение данного многообразия $(X, \omega)$ возникает при фиксации той же функции. Таким образом определяется отображение из пространства $C^{\infty}\left(\mathbb{C P}^{1}, \mathbb{R}\right)$ в пространство всевозможных лагранжевых слоений на $(X, \omega)$, включая, конечно, и особые. 
Во всех примерах настоящей работы торическая геометрия является базовой, так как даже неторические многообразия Фано, рассматриваемые нами, представляют собой полные пересечения в главном торическом многообразии - проективном пространстве. Напомним, что торическая структура на симплектическом многообразии $(X, \omega)$ задается набором отображений моментов $\left(f_{1}, \ldots, f_{n}\right)$ со значениями в выпуклом многограннике $P_{n} \subset \mathbb{R}^{n}$, и, как было доказано в работе [4], всегда существует комплексное торическое многообразие с обильным линейным расслоением, которое симплектически изоморфно $(X, \omega)$. Это влечет существование соответствующей согласованной кэлеровой структуры, так что функции $f_{i}$ являются квантуемыми в смысле Березина-Ронсли [5], т.е. их гамильтоновы векторные поля сохраняют кэлерову структуру. Согласно геометрической формулировке квантовой механики [6] эти вещественные гладкие функции могут рассматриваться как квантовые наблюдаемые (называемые символами Березина).

Инфинитезимальное гамильтоново действие каждой такой функции на голоморфных объектах над $X$ индуцирует инфинитезимальную симметрию таких объектов. Поэтому если взять проективизации $\mathbb{P}\left(H^{0}\left(X, K_{X}^{-k}\right)\right)$ пространств голоморфных сечений степеней антиканонического расслоения $K_{X}^{-1}$, то каждая функция $f_{i}$ будет генерировать некоторое гамильтоново векторное поле $\Theta_{f_{i}}^{k}$ на $\mathbb{P}\left(H^{0}\left(X, K_{X}^{-k}\right)\right)$. При этом дивизор $D \in|-k K|$ является инвариантным относительно $X_{f_{i}}$, если и только если $\Theta_{f_{i}}^{k}$ обращается в нуль в соответствующей точке $p_{D}$. Поэтому в терминах индуцируемой гладкой функции на соответствующем проективном пространстве $\mathbb{P}\left(H^{0}\left(X, K_{X}^{-k}\right)\right)$ инвариантный пучок из полной линейной системы $|-k K|$ существует, если и только если $F_{i, k}$ обладает критической проективной прямой. Такое возможно, если размерность проективного пространства больше $\operatorname{dim} X$.

Поскольку мы работаем с антиканоническим классом и его степенями, видно, что торическое многообразие Фано должно обладать псевдоторической структурой над проективной прямой. Эта прямая может быть использована в исследованиях свойства сдвигаемости для стандартных слоев торических слоений, являющихся лагранжевыми торами. Для этого можно воспользоваться следующей схемой: фиксируем подходящий набор отображений моментов $\left(f_{1}, \ldots, f_{n}\right)$, соответствующих стандартной торической структуре, удаляем одно из них, скажем, $f_{n}$, и затем находим пучок дивизоров, инвариантных относительно гамильтонова действия $f_{1}, \ldots, f_{n-1}$. Условие Фано влечет, что такой пучок существует, и поэтому начальные данные $\left(f_{1}, \ldots, f_{n}\right)$ могут быть заменены на данные вида $\left(f_{1}, \ldots, f_{n-1}, \psi\right)$. В то же время эти новые данные согласуются с исходными: можно показать, что найдется гладкая функция $h_{0} \in C^{\infty}\left(\mathbb{C P}^{1}, \mathbb{R}\right)$ такая, что индуцируемое ею лагранжево слоение на $X$ то же, что и задаваемое набором $\left(f_{1}, \ldots, f_{n}\right)$. Это замечание показывает, что всякий стандартный лагранжев тор из торического слоения может быть сведен шаг за шагом к цепочке гладких петель на проективных прямых, так что гамильтоновы инварианты этого тора могут быть выражены в терминах симплектических площадей, ограничиваемых этими петлями.

Гладкая петля разбивает проективную прямую на две части. Петля называется меридиональной, если эти части имеют одинаковую симплектическую площадь. 
Известно, что гладкая петля является несдвигаемой, если и только если она меридиональная. На основании этого можно заметить, что гладкий слой торического слоения торического многообразия Фано является несдвигаемым, только если все петли, получаемые с помощью такой редукции, являются меридиональными. Остается выразить гамильтоновы инварианты (периоды) этого слоя через разности площадей для каждой петли, чтобы установить следующий факт: если $S$ - гладкий лагранжев тор, являющийся слоем торического слоения торического многообразия Фано $X$, то $S$ является несдвигаемым только тогда, когда $S$ бор-зоммерфельдов относительно антиканонического класса.

Ниже этот факт доказан для торов Клиффорда в проективном пространстве (предложения 1 и 2) с помощью идей, приходящих из псевдоторических рассмотрений. Подчеркнем, что сам по себе этот факт в случае торов Клиффорда очевиден, однако наш инвариантный метод допускает обобщения на нетривиальный случай и показывает, что существуют псевдоторические структуры на проективных пространствах. Таким образом, доказана малая часть гипотез, представленных в статье [7]. В полной общности этот факт может быть доказан тем же методом. Мы планируем сделать это в следующей работе, в которой главное внимание будет уделено общему псевдоторическому многообразию Фано, а торический случай будет простым следствием такого общего случая.

Вторая половина настоящей работы посвящена случаю неторических многообразий Фано, допускающих существование псевдоторической структуры. Сначала мы доказываем, что псевдоторическое многообразие Фано может быть расслоено на изотропные подмногообразия так, что общим слоем будет гладкий лагранжев тор (теорема 1). Таким образом, псевдоторическая структура схожа с торической в том, что она дает лагранжевы слоения. Затем мы доказываем, что любая гладкая неприводимая квадрика допускает псевдоторическую структуру (теорема 2). Напомним, что квадрика есть торическое многообразие только в размерностях 1 и 2 . В конце работы мы обсуждаем, как псевдоторические структуры могут быть использованы при построении специальных лагранжевых слоений многообразий Фано, введенных недавно в работе [8].

План статьи таков. Раздел 1 содержит предварительные конструкции, не являющиеся необходимыми для остальной части работы, но объясняющие, почему мы понимаем отображения моментов как символы Березина, а лагранжевы торы - как квантовые состояния. Раздел 2 начинается с конструкции и вычислений для проективной плоскости. Мы показываем, что если клиффордов тор $\mathbb{C P}^{2}$ не является бор-зоммерфельдовым относительно антиканонического класса, то он сдвигаем. Затем та же стратегия используется для установления аналогичного факта в любой размерности. Далее в качестве следствия показано различие торов типа Клиффорда и типа Чеканова. Случай не торических, но псевдоторических многообразий Фано изучается в разделе 3. Здесь сначала мы рассматриваем примеры, далее даем определение псевдоторической структуры и доказываем, что гладкая квадрика в любой размерности обладает такой структурой. В качестве приложения мы приводим лагранжево слоение трехмерной квадрики, являющееся специальным в смысле работы [8]. 


\section{1. ОТ СИМВОЛОВ БЕРЕЗИНА К ЛАГРАНЖЕВЫМ СЛОЕНИЯМ}

Квантовая механика может быть сформулирована в геометрических терминах [6], более точно - в терминах алгебраической геометрии. Как уже неоднократно обсуждалось [5], фазовое пространство квантово-механической системы представляется алгебраическим многообразием, снабженным кэлеровой метрикой ходжева типа. При этом квантовые наблюдаемые представляются вещественными гладкими функциями, гамильтоновы векторные поля которых сохраняют и комплексную, и симплектическую структуры, т.е. ими представлены инфинитезимальные симметрии кэлеровой структуры. Такие функции в книге [6] названы символами. Главным примером является проективное пространство со стандартной метрикой ФубиниШтуди $g_{\mathrm{FS}}$; в этом случае любой символ получается из соответствующего самосопряженного оператора в гильбертовом пространстве, проективизация которого есть наше проективное пространство. Для восстановления этого самосопряженного оператора по данным $\left(\mathbb{C P}^{n}, \omega, g_{\mathrm{FS}}\right)$, где $\omega$ - соответствующая $g_{\mathrm{FS}}$ кэлерова форма, к символу $f$ применяется один из методов геометрического квантования, а именно метод Березина-Ронсли. Так как гамильтоново действие функции $f$ сохраняет кэлерову структуру, это действие может быть продолжено на пространство сечений линейного расслоения $\mathcal{O}(1)$ путем выбора эрмитовой связности с формой кривизны, пропорциональной симплектической форме. Тогда соответствующий оператор Сурьо-Костанта $A_{f}$ сохраняет подпространство голоморфных сечений $\mathcal{O}(1)$, они и будут самосопряженным оператором и гильбертовым пространством (см., например, работу [5]).

Таким образом, для данного проективного пространства $\mathbb{C P}^{n}$ пространство символов полностью описано; нетрудно видеть, что оно является подалгеброй Пуассона в $C^{\infty}\left(\mathbb{C} \mathbb{P}^{n}, \mathbb{R}\right)$ относительно скобок Пуассона, определяемых симплектической формой $\omega$. Каждый (невырожденный) символ $f$ включается в некоторую коммутативную подалгебру алгебры Пуассона, натянутую на набор символов $f=\left(f_{1}, \ldots, f_{n}\right)$. Заметим, что это множество приходит из множества коммутирующих самосопряженных операторов; максимальное число таких операторов равно $n+1$, но, поскольку единичный оператор переходит в постоянную функцию при проективизации, ранг множества коммутирующих алгебраически независимых почти всюду символов равен $n$. Для проективного пространства эти символы являются отображениями моментов на языке торической геометрии.

Распространим наше рассмотрение на более широкий класс алгебраических многообразий. Пусть $X$ - такое многообразие и $\omega$ - кэлерова форма метрики ходжева типа на $X$. Тогда нетрудно видеть, что существование нетривиального символа на $X$ возможно, если и только если выполнено следующее условие: возьмем соответствующее обильное линейное расслоение $L \rightarrow X$, рассмотрим вложение $\phi: X \rightarrow\left(\mathbb{P}\left(H^{0}\left(X, L^{k}\right)^{*}\right)\right.$, при этом найдется символ $f$ на $\mathbb{P}\left(H^{0}\left(X, L^{k}\right)^{*}\right)$ такой, что гамильтоново векторное поле $X_{f}$ касательно образу $\phi(X)$ в каждой его точке. Это предположение упрощает задачу поиска алгебраических многообразий с символами, т.е. всех возможных квантовых фазовых пространств обобщенной в смысле Аште- 
кара квантовой механики. Все такие многообразия являются подмногообразиями проективного пространства $\mathbb{C P}^{n}$, параллельными гамильтоновым векторным полям некоторых символов.

В такой формулировке задача исследовалась Белевым [9]. Например, в случае пространства $\mathbb{C P}^{2}$ он установил, что гладкая кривая $C \subset \mathbb{C P}^{2}$ допускает существование символа, если и только если она рациональна. При этом возникает интересный эффект - если гамильтоново действие некоторого символа $f$ сохраняет невырожденную конику, то существует целый пучок коник (включающий в себя начальную), инвариантных относительно гамильтонова действия того же символа. И это общий принцип для неприводимых дивизоров в проективных пространствах [9].

В самом деле, пусть $D$ - неприводимый дивизор в $\mathbb{C P}^{n}$ и $f$ - символ, сохраняющий $D$. Так как каждый символ соответствует самосопряженному оператору в $\mathbb{C}^{n+1}$, найдется система однородных координат $\left[z_{0}: \ldots: z_{n}\right]$, относительно которой $f$ имеет следующую "диагональную" форму:

$$
f=\frac{\sum_{i=0}^{n} \lambda_{i}\left|z_{i}\right|^{2}}{\sum_{i=0}^{n}\left|z_{i}\right|^{2}},
$$

где $\lambda_{i}$ - критические значения $f$. Поскольку $D$ неприводим, в этих координатах он определен многочленом $P(z)$, состоящим не менее чем из двух слагаемых, при этом, поскольку $D$ инвариантен, сумма весов $\lambda_{i}$ для каждого слагаемого есть константа. Отсюда видно, что для любой линейной комбинации тех же слагаемых множество нулей соответствующего полинома обязано быть инвариантным дивизором. Отсюда следует, что если имеется инвариантный неприводимый дивизор, то имеется и пучок инвариантных дивизоров. Заметим, что в этом рассуждении мы использовали торическую структуру на $\mathbb{C P}^{n}$, что было важно, так как произвольный символ априори не порождает $U(1)$-действие на данном алгебраическом многообразии, не дает замкнутых орбит и т.д., в то время как отображение моментов должно обладать этими свойствами по определению. Но это подсказывает, что обобщение конструкций слоений на лагранжевы торы на случай неторических многообразий Фано должно следовать почти по той же схеме: если $X$ допускает набор коммутирующих символов $\left(f_{1}, \ldots, f_{n-1}\right)$ и не допускает полного набора, будучи неторическим, зато допускает инвариантный пучок, то лагранжевы слоения могут быть построены. Например, эту схему можно приложить к случаю неторической поверхности дель Пеццо $\mathbb{C P}_{4}^{2}[10]$.

Вернемся к случаю проективной плоскости. В работе [3] был представлен следующий пример. Пучок $\mathbb{C P}_{\alpha, \beta}^{1}$ на $\mathbb{C P}^{2}$ задан уравнением

$$
\alpha z_{0}^{2}+\beta z_{1} z_{2}=0
$$

символ в координатах на гильбертовом пространстве $\mathbb{C}^{3}$ соответствует диагональному оператору с собственными значениями $(1,0,-2)$. В однородных координатах $\left[z_{0}: z_{1}: z_{2}\right]$ эта функция имеет вид

$$
f=\frac{\left|z_{0}\right|^{2}-2\left|z_{2}\right|^{2}}{|z|^{2}},
$$

где $|z|^{2}=\sum_{i=0}^{2}\left|z_{i}\right|^{2}$, как обычно. 
Базисное множество пучка $\mathbb{C P}_{\alpha, \beta}^{1}$ содержится в критическом множестве функции $f$, и особые слои нашего пучка содержат все критические точки функции $f$; как мы увидим ниже, это общий принцип для инвариантных симплектических пучков. Пучок $\mathbb{C P}_{\alpha, \beta}^{1}$ содержит две особые коники: $D_{1: 0}$ - двойную прямую, $D_{0: 1}$ - пару прямых, так что мы имеем две выделенные точки на $\mathbb{C P}_{\alpha, \beta}^{1}$ с координатами $[1: 0]$ и $[0: 1]$. Рассмотрим сначала гладкую функцию $h$ на $\mathbb{C P}_{\alpha, \beta}^{1}$, задаваемую уравнением

$$
h=\frac{|\alpha|^{2}-|\beta|^{2}}{|\alpha|^{2}+|\beta|^{2}}
$$

и являющуюся символом на $\mathbb{C P}_{\alpha, \beta}^{1}$. Она имеет в точности две критические точки, соответствующие двум вырожденным элементам пучка. Как было показано в работе [3], такая функция $h$ дает нам стандартное клиффордово слоение на $\mathbb{C P}^{2} . \mathrm{B}$ самом деле, гладкое множество уровня

$$
\gamma_{c}=\{h=c\} \subset \mathbb{C P}_{\alpha, \beta}^{1}, \quad-1<c<1,
$$

определено условиями $|\alpha|=$ const, $|\beta|=$ const, и из уравнения пучка (1) следует, что соответствующие множества на $\mathbb{C P}^{2}$ выделены условиями Клиффорда $\left|z_{i}\right|=$ const. Таким образом, рассмотренная выше функция $h$ просто восстанавливает торическое лагранжево слоение.

Рассмотрим теперь другую функцию $h$, заданную равенством

$$
h=\frac{|\beta|^{2}-|a \alpha-\beta|^{2}}{|\beta|^{2}+|a \alpha-\beta|^{2}},
$$

где $a$ - положительный вещественный параметр. Эта функция снова имеет две критические точки, которые на этот раз соответствуют следующим элементам пучка: снова двойной прямой $2 l=\left\{z_{0}^{2}=0\right\}$ и невырожденной конике $Q=\left\{z_{0}^{2}=-a z_{1} z_{2}\right\}$. Поэтому получаемое в результате слоение пространства $\mathbb{C P}^{2}$ размерностно вырождается на приводимой кубической кривой $l \cup D$ и содержит один особый тор. Это лагранжево слоение есть в точности неторическое слоение, построенное Ору [8]. Лагранжевы торы, получаемые при этом, разделены на два типа [8]: тип Клиффорда и тип Чеканова. Отличие определяется проекцией на $\mathbb{C P}_{\alpha, \beta}^{1}$ : тор, соответствующий петле $\gamma_{c}$ с отрицательным $c$, есть тор типа Клиффорда; если $c$ положительно, то соответствующий тор имеет тип Чеканова. Эти типы различают классы эквивалентности по модулю гамильтоновой изотопии.

Все наши конструкции являются обобщением конструкции Ору. А она, как оказалось, может быть обобщена на случай произвольного торического многообразия Фано, что является главным результатом работы [3].

\section{2. ТОРЫ КЛИФФОРДА И ЧЕКАНОВА В $\mathbb{C P}^{2}$ И $\mathbb{C P}^{n}$}

Рассмотрим проективное пространство $\mathbb{C P}^{2}$ со стандартной метрикой ФубиниШтуди и соответствующей симплектической формой $\omega$. Таким образом, $\mathbb{C P}^{2}$ снабжено эрмитовой тройкой и тем же снабжено его касательное расслоение. Следовательно, его детерминант $-K$ снабжен соответствующей эрмитовой структурой, 
и можно рассмотреть пространство эрмитовых связностей $\mathcal{A}_{\mathrm{h}}(-K)$. Так как канонический класс пропорционален классу $[\omega]$, имеется выделенная орбита в $\mathcal{A}_{\mathrm{h}}(-K)$, определяемая условием пропорциональности формы кривизны симплектической форме. Возьмем произвольную связность $а$ из этой орбиты и рассмотрим ограничение пары $(-K, a)$ на произвольный лагранжев тор $S \subset \mathbb{C P}^{2}$. Говорят, что тор бор-зоммерфельдов относительно антиканонического класса, если это ограничение допускает ковариантно постоянное сечение. Это определение имеет смысл для любого монотонного симплектического многообразия, например для произвольного многообразия Фано [11].

Напомним, что лагранжево подмногообразие $S$ является сдвигаемым, если найдется гамильтонова изотопия $\phi_{t}$ такая, что $S \cap \phi_{t}(S)=\varnothing$ для некоторого $t$. В противном случае оно называется несдвигаемым.

Согласно одной из гипотез, представленных в статье [7], регулярный слой торического слоения торического многообразия Фано несдвигаем, если и только если он является бор-зоммерфельдовым относительно антиканонического класса. Утверждение очевидно в случае пространства $\mathbb{C P}^{n}$, но для дальнейших приложений мы приводим подробное доказательство, основанное на конструкции с инвариантным пучком из предыдущего раздела.

ПредлоЖеНИЕ 1. Несдвигаемый клиффордов тор в $\mathbb{C P}^{2}$ является бор-зоммерфельдовым относительно антиканонического класса.

ДоказАтельство. Обратимся к рассуждениям из раздела 1. Регулярный клиффордов тор $S \subset \mathbb{C P}^{2}$ задается условиями $f=c_{1}, h=c_{2}$, причем $c_{1}$ не является критическим значением для $f$, т.е. $c_{1} \neq-2,1$, а $c_{2}$ не является критическим значением для $h$, т.е. $c_{2} \neq-1,1$, где функции $f$ и $h$ заданы в (2) и (3). Заметим, что единственный бор-зоммерфельдов относительно антиканонического класса слой соответствует значениям $c_{1}=0, c_{2}=0$. Предположим, что слой не является бор-зоммерфельдовым относительно антиканонического класса; обозначим его как $S_{c_{1}, c_{2}}$. Тогда мы можем утверждать, что если $c_{2} \neq 0$, т.е. линия уровня $\gamma_{c_{2}}=\left\{h=c_{2}\right\} \subset \mathbb{C P}_{\alpha, \beta}^{1}$ не является меридиональной, то найдется гамильтоново преобразование $\phi_{t}$ плоскости $\mathbb{C P}^{2}$, которое перемещает $S_{c_{1}, c_{2}}$ в некоторое $\phi_{t}\left(S_{c_{1}, c_{2}}\right)$ так, что

$$
S_{c_{1}, c_{2}} \cap \phi_{t}\left(S_{c_{1}, c_{2}}\right)=\varnothing .
$$

Для доказательства этого утверждения мы явно построим вещественную гладкую функцию $F$ на $\mathbb{C P}^{2}$, гамильтоново векторное поле которой порождает необходимое преобразование.

Базисное множество пучка (1) состоит из двух критических точек $f$, а именно $[0: 1: 0]$ и $[0: 0: 1]$, и пучок содержит особую конику с особенностью в $[1: 0: 0]$. Зафиксируем достаточно малое число $\varepsilon$ так, что

$$
\varepsilon \ll \min \left(\left|c_{1}-1\right|,\left|c_{1}\right|,\left|c_{1}-2\right|\right) .
$$

Тогда шары радиуса $\varepsilon$ с центрами в $[1: 0: 0],[0: 1: 0]$ и $[0: 0: 1]$ не пересекают наш тор $S_{c_{1}, c_{2}}$. Обозначим эти шары как $B_{1}, B_{2}$ и $B_{3}$. Рассмотрим голоморфное 
отображение

$$
\psi: \mathbb{C P}^{2}-B \rightarrow \mathbb{C P}_{\alpha, \beta}^{1},
$$

определяемое пучком (1), где $B$ - базисное множество пучка:

$$
B=[0: 1: 0] \cup[0: 0: 1] .
$$

Тогда любая гладкая вещественная функция $h_{\mathrm{c}}: \mathbb{C P}_{\alpha, \beta}^{1} \rightarrow \mathbb{R}$ может быть поднята на $\mathbb{C P}^{2}-\left(B_{1} \cup B_{2}\right)$ а затем продолжена до гладкой функции $F_{h_{\mathrm{c}}}$ на всем $\mathbb{C P}^{2}$. Для этого возьмем шары $B_{1}^{\prime}, B_{2}^{\prime}, B_{3}^{\prime}$ с теми же центрами с радиусом $\varepsilon / 2$ и определим $F=F_{h_{\mathrm{c}}}$ на пяти разных частях $\mathbb{C P}^{2}$ следующим образом:

$$
\begin{aligned}
& F=\psi^{*} h_{\text {с на }} \mathbb{C P}^{2}-\left(B_{1} \cup B_{2} \cup B_{3}\right) ; \\
& F=0 \text { на } B_{i}^{\prime}, i=1,2,3 ;
\end{aligned}
$$

на $B_{i}-B_{i}^{\prime}$ функция $F$ линейно убывает с большой границы $\partial B_{i}$ до малой границы $\partial B_{i}^{\prime}$ так, что $F$ - гладкая функция на $\mathbb{C P}^{2}$.

Гамильтоново векторное поле данной функции $F$ симплектически ортогонально "центральным неособым частям" слоев, если определить такую центральную часть слоя $D_{p}$ как

$$
D_{p}^{\mathrm{c}}=D_{p}-\left(D_{p} \cap\left(B_{1} \cup B_{2} \cup B_{3}\right)\right) .
$$

Заметим, что мы должны вырезать $B_{1}$, поскольку гамильтоново векторное поле функции $\psi^{*} h_{\mathrm{c}}$ имеет особенность в $[1: 0: 0]$.

Таким образом, гамильтоново действие $\phi_{t}(F)$ передвигает "центральные неособые части" гладких слоев в "центральные неособые части" гладких слоев и закручивает окрестности $B_{1}$ и $B_{2}$ базисного множества. Поэтому круговое движение снизу, порождаемое морсовской функцией $h_{\mathrm{c}}$ с двумя критическими точками на $\mathbb{C P}_{\alpha, \beta}^{1}$, поднимается до "квазикругового" действия, которое закручивает окрестности базисного множества и особой точки, оставляя их неподвижными, и меняет местами "центральные неособые части" $D_{p}^{\text {c } г л а д к и х ~ с л о е в . ~}$

Определим теперь функцию $h_{\mathrm{c}}$ на $\mathbb{C P}_{\alpha, \beta}^{1}$, которая порождает нужную нам трансформацию. Такая функция имеет вид

$$
h_{\mathrm{c}}=\frac{2 \operatorname{Re} \alpha \bar{\beta}}{|\alpha|^{2}+|\beta|^{2}} ;
$$

у нее есть две критические точки на $\mathbb{C P}_{\alpha, \beta}^{1}$ с координатами $[1: 1]$ и $[1:-1]$, и соответствующее гамильтоново движение, индуцируемое $X_{h_{\mathrm{c}}}$ на $\mathbb{C P}_{\alpha, \beta}^{1}$, есть вращение с фиксированными полюсами $[1: 1]$ и $[1:-1]$. Это вращение в момент времени $t=\pi$ переводит петлю $\gamma_{c_{2}}=\left\{h=c_{2}\right\}$ в "зеркальную" петлю $\gamma_{-c_{2}}=\left\{h=-c_{2}\right\}$, которая не пересекает $\gamma_{c_{2}}$, если $\gamma_{c_{2}}$ не является меридиональной.

Эти аргументы применимы и к функции $F$; гамильтоново движение $\phi_{t}$, индуцируемое $X_{F}$ на $\mathbb{C P}^{2}$, перемещает слои в слои за исключением частей, близких или к базисному множеству, или к особой точке. Отсюда следует, что наш лагранжев тор $S_{c_{1}, c_{2}}$ перемещается посредством $\phi_{\pi}$ в тор $S_{c_{1},-c_{2}}$, а последний не пересекает исходный, если только $c_{2}$ не равно нулю. Заметим, что условие $c_{2}=0$ эквивалентно тому, что $|\alpha|=|\beta|$. 
С другой стороны, можно рассмотреть любую перестановку координат $z_{0}, z_{1}, z_{2}$ и взять другой символ и другой пучок, например

$$
\alpha^{\prime} z_{1}^{2}+\beta^{\prime} z_{0} z_{2}=0
$$

после чего повторить рассуждения для этих данных. Снова слой оказывается сдвигаемым, если он отвечает линии уровня соответствующей функции с $\left|\alpha^{\prime}\right| \neq\left|\beta^{\prime}\right|$. Каждый тор Клиффорда однозначно определен двумя линиями уровня на $\mathbb{C P}_{\alpha, \beta}^{1}$ и $\mathbb{C P}_{\alpha^{\prime}, \beta^{\prime}}^{1}$, таким образом, представленные выше аргументы показывают, что если тор Клиффорда несдвигаем, то он задается условиями

$$
z_{0}^{2}=e^{i \phi_{1}} z_{1} z_{2}, \quad z_{1}^{2}=e^{i \phi_{2}} z_{0} z_{2}
$$

где $\phi_{i}$ - вещественные параметры. И, как показано в работе [7], такой тор является в точности бор-зоммерфельдовым относительно антиканонического класса. Предложение доказано.

С другой стороны, в той же работе [7] показано, что бор-зоммерфельдов слой в этом случае является монотонным, отсюда имеем

СлЕДСТВИЕ 1. Если тор Клиффорда несдвигаем, то он монотонен.

Рассмотрим теперь случай пространства $\mathbb{C P}^{n}$ при $n>2$. Представим ту же схему рассуждений для доказательства следующего утверждения.

ПреДЛОЖениЕ 2. Пусть $S$ - клифбордов тор в $\mathbb{C P}^{n}$. Тогда он несдвигаем, только если он является бор-зоммерфелъдовым относителъно антиканонического класса.

ДокАЗАтЕльство. Возьмем пространство $\mathbb{C P}^{n}$ и рассмотрим набор интегралов $\left(f_{1}, \ldots, f_{n}\right)$, определяющих переменные действия в соответствующем слоении Клиффорда. Отображение действия

$$
\left(f_{1}, \ldots, f_{n}\right): \mathbb{C P}^{n} \rightarrow P_{n} \subset \mathbb{R}^{n}
$$

определяет образ $P_{n}$, задаваемый условиями

$$
x_{1}+\cdots+x_{n}=1, \quad x_{i} \geqslant 0,
$$

и для соответствующего универсального базиса в $H_{1}\left(T^{n}, \mathbb{Z}\right)$ значения $x_{1}, \ldots, x_{n}$ равны периодам соответствующих петель относительно симплектической формы. Тор Клиффорда является бор-зоммерфельдовым относительно канонического класса, если и только если его периоды представлены целыми числами по модулю $1 /(n+1)$. Отсюда видно, что существует единственный тор, бор-зоммерфельдов относительно антиканонического класса, в размерности $n$ (но имеется много изотропных торов). Заметим, что $f_{i}$ вырождены как символы в нашей терминологии, поскольку каждый $f_{i}$ имеет неизолированные критические точки, составляющие дивизоры.

Зафиксируем систему однородных координат $\left[z_{0}: \ldots: z_{n}\right]$ так, чтобы каждый $f_{i}$ вырождался на гиперплоскости $\left\{z_{i}=0\right\}$. Исключим один символ, например $f_{n}$, 
из набора $\left(f_{1}, \ldots, f_{n}\right)$. Случаи четного и нечетного $n$ различаются тем, что мы рассматриваем разные пучки и символы.

Если $n$ нечетно, то в системе координат $\left[z_{0}: \ldots: z_{n}\right]$ рассматривается пучок

$$
\left\{D_{u}\right\}=\left\{u_{0} z_{0} \ldots z_{(n-1) / 2}+u_{1} z_{(n+1) / 2} \ldots z_{n}=0\right\}
$$

задающий комплексное отображение

$$
\psi: \mathbb{C P}^{n}-B \rightarrow \mathbb{C P}_{u}^{1},
$$

где $B$ - базисное множество пучка, составленное из $((n+1) / 2)^{2}$ гиперплоскостей комплексной размерности $n-2$. Набор символов (отображений моментов), сохраняющих элементы пучка $\left\{D_{u}\right\}$, выделен условием

$$
\lambda_{0}+\cdots+\lambda_{(n-1) / 2}=\lambda_{(n+1) / 2}+\cdots+\lambda_{n}
$$

наложенным на критические значения $\lambda_{i}$.

Если $n$ четно, ты мы выбираем пучок

$$
\left\{D_{u}\right\}=\left\{u_{0} z_{0}^{2} z_{1} \ldots z_{n / 2-1}+u_{1} z_{n / 2} \ldots z_{n}\right\}
$$

а символы должны удовлетворять условию

$$
2 \lambda_{0}+\lambda_{1}+\cdots+\lambda_{n / 2-1}=\lambda_{n / 2}+\cdots+\lambda_{n}
$$

Далее оба случая можно рассматривать вместе.

Очевидно, что наши исходные символы $f_{1}, \ldots, f_{n-1}$ можно с помощью некоторого линейного преобразования заменить набором невырожденных символов $f_{1}^{\prime}, \ldots, f_{n-1}^{\prime}$ так, что каждый символ $f_{i}^{\prime}$ удовлетворяет (8а) или (8б) в зависимости от четности $n$ и, следовательно, каждый символ $f_{i}^{\prime}$ сохраняет каждый элемент в $\left\{D_{u}\right\}$. Симплекс вырождения $\Delta_{n-1} \subset \mathbb{C P}^{n}$ набора $\left(f_{1}^{\prime}, \ldots, f_{n-1}^{\prime}\right)$ состоит из $(n+1) n / 2$ гиперплоскостей размерности $n-2$. Общий слой $\psi$ гладкий, причем имеются в точности два особых слоя, проектирующихся в точки $[0: 1]$ и $[1: 0]$ в $\mathbb{C P}_{u}^{1}$. Рассмотрим множества особенностей слоев $D_{[1: 0]}$ и $D_{[0: 1]}$, обозначим их через $\operatorname{Sing}_{0}$ и $\operatorname{Sing}_{1}$. Нетрудно видеть, что

$$
\Delta_{n-1}=B \cup \operatorname{Sing}_{0} \cup \operatorname{Sing}_{1} .
$$

Выберем функцию $h$ на $\mathbb{C P}_{u}^{1}$ вида (3). Мы утверждаем, что слоение $\mathbb{C P}^{n}-B$, определяемое $\left(f_{1}^{\prime}, \ldots, f_{n-1}^{\prime}, \psi^{*} h\right)$, совпадает со слоением Клиффорда. В самом деле, возьмем клиффордов тор $T_{\left(c_{1}, \ldots, c_{n}\right)}$, определяемый условиями $\left|z_{i}\right|^{2}=c_{i}$, так что $c_{i}-$ регулярные значения отображений моментов $f_{1}, \ldots, f_{n}$ (поэтому они должны быть положительными). Так как $f_{j}^{\prime}$ получаются линейным преобразованием $f_{i}$, это же преобразование переводит набор $\left(c_{1}, \ldots, c_{n-1}\right)$ в значения $f_{j}^{\prime}$, которые мы обозначаем как $\left(c_{1}^{\prime}, \ldots, c_{n-1}^{\prime}\right)$. Значение $h$ равно

$$
c=\frac{c_{(n+1) / 2} \ldots c_{n}}{c_{1} \ldots c_{(n-1) / 2}}-1 \quad \text { или } \quad c=\frac{c_{n / 2} \ldots c_{n}}{c_{1} \ldots c_{n / 2-1}}
$$


в четном или нечетном случаях соответственно. Тогда очевидным образом

$$
S_{\left(c_{1}^{\prime}, \ldots, c_{n-1}^{\prime}, c\right)}=\left\{f_{j}^{\prime}=c_{j}^{\prime}, \psi^{*} h=c\right\}=T_{\left(c_{1}, \ldots, c_{n}\right)} .
$$

Следовательно, без ограничения общности мы можем приписать клиффородовым торам числа $\left(c_{1}^{\prime}, \ldots, c_{n-1}^{\prime}, c\right)$ вместо $\left(c_{1}, \ldots, c_{n}\right)$.

Следующим шагом является построение такой гладкой функции $F$ на всем $\mathbb{C P}^{n}$, гамильтоново векторное поле которой индуцировало бы движение клиффордова тоpa $T_{\left(c_{1}^{\prime}, \ldots, c_{n-1}^{\prime}, c\right)}$ в клиффордов тор $T_{\left(c_{1}^{\prime}, \ldots, c_{n-1}^{\prime},-c\right)}$. Это означало бы, что клиффордов тор несдвигаем, только если он проецируется отображением $\psi$ в меридиональную петлю на $\mathbb{C P}_{u}^{1}$.

Такая функция $F$ строится тем же способом, что и при доказательстве предложения 1. Выберем радиус $\varepsilon$ малой окрестности $\mathcal{O}_{1}\left(\Delta_{n-1}\right)$ множества вырождения $\Delta_{n-1}$ так, чтобы $T_{\left(c_{1}^{\prime}, \ldots, c_{n-1}^{\prime}, c\right)}$ не пересекал $\mathcal{O}_{1}\left(\Delta_{n-1}\right)$, и возьмем меньшую окрест-

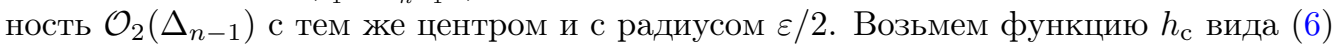
на $\mathbb{C P}_{u}^{1}$ и построим глобальную функцию $F$ на $\mathbb{C P}^{n}$ по уже знакомым правилам:

$F$ обращается в нуль внутри $\mathcal{O}_{2}\left(\Delta_{n-1}\right)$;

$F$ равна $\psi^{*} h_{\mathrm{c}}$ вне $\mathcal{O}_{1}\left(\Delta_{n-1}\right)$;

$F$ гладко убывает от границы $\mathcal{O}_{1}\left(\Delta_{n-1}\right)$ до границы $\mathcal{O}_{2}\left(\Delta_{n-1}\right)$.

Свойства такой функции $F$ те же, что свойства функции из доказательства предложения 1: ее гамильтоново действие переводит “центральные части” слоев в “центральные части", следовательно, она индуцирует гамильтонову изотопию тора $T_{\left(c_{1}^{\prime}, \ldots, c_{n-1}^{\prime}, c\right)}$ в тор $T_{\left(c_{1}^{\prime}, \ldots, c_{n-1}^{\prime},-c\right)}$, что и декларировалось выше.

Таким образом, тор Клиффорда несдвигаем, только если $c=0$ и, следовательно,

$$
c_{1} \ldots c_{(n-1) / 2}=c_{(n+1) / 2} \ldots c_{n}
$$

если $n$ нечетно, или

$$
c_{1} \ldots c_{n / 2-1}=c_{n / 2+1} \ldots c_{n}
$$

в случае четного $n$. Но мы с самого начала могли исключить другую $f_{i}$ из набора и провести те же самые рассуждения. Отсюда получаем, что все $c_{i}$ обязаны быть равными, и это означает, что каждый несдвигаемый клиффордов тор обязан быть бор-зоммерфельдовым относительно антиканонического класса. Этим завершается доказательство предложения.

Итог обсуждения клиффордовых торов в $\mathbb{C P}^{n}$ подводится следующим следствием.

СлЕДСТВИЕ 2. Каждый несдигаемый клифбордов тор в $\mathbb{C P}^{n}$ монотонен.

ЗАмЕчание 1. В работе [8] представлены два типа лагранжевых торов в $\mathbb{C P}^{2}$, тип Клиффорда и тип Чеканова, причем эти торы разделены особым тором в слоении Ору. В свете работы [3] мы можем указать на то, что торы типа Чеканова возникают, если функция $h$ на $\mathbb{C P}_{\alpha, \beta}^{1}$ имеет две критические точки $p_{\max }, p_{\min } \in \mathbb{C P}_{\alpha, \beta}^{1}$, так что только одна из них подлежит особому элементу пучка, а именно двойной 
прямой $\left\{z_{0}^{2}=0\right\}$. Тогда имеются не две, а три выделенные точки $p_{\max }, p_{\min }, p_{\operatorname{sing}}$ в $\mathbb{C P}_{\alpha, \beta}^{1}$, последняя из которых подлежит особому слою $\left\{z_{1} z_{2}=0\right\}$, как в случае $h$ вида (4). Как показано в работе [3], произвольная морсовская функция на $\mathbb{C P}_{\alpha, \beta}^{1}$ задает лагранжево слоение, слои которого проецируются при отображении (5) в линии уровня этой функции. Множество линий уровня данной функции $h$ разделено точкой $p_{\text {sing }}$, и торы из разных камер имеют типы Клиффорда и Чеканова. Конструкция с инвариантным пучком на $C \mathbb{P}^{2}$ показывает, что в данной ситуации торы Клиффорда и Чеканова могут быть переведены друг в друга посредством симплектоморфизма, но не гамильтоновой изотопии. В самом деле, для любого вращения проективной прямой $\mathbb{C P}_{\alpha, \beta}^{1}$ имеется соответствующее закручивание слоев $\psi$, что дает некоторый симплектоморфизм $\mathbb{C P}^{2}$. Но особая точка $[1: 0: 0]$ является особенностью поднятого гамильтонова векторного поля, поэтому такой скручивающий симплектоморфизм не является гамильтоновой изотопией. В итоге получаем, что торы Клиффорда и торы Чеканова с одними и теми же гамильтоновыми инвариантами являются симплектоформными, но не изотопными.

Можно определить торы Клиффорда и Чеканова и в случае $\mathbb{C P}^{n}$, используя инвариантный пучок из доказательства предложения 2 и функцию $h$ вида (4). Кроме того, из того же доказательства проистекает следующее замечание. Рассмотрим произвольный однородный полином $P(z)$ степени $d$ от переменных $z=\left(z_{0}, \ldots, z_{n}\right)$. Тогда $P(z)$ задает некоторое отображение

$$
\psi: \mathbb{C P}^{n}-B \rightarrow \mathbb{C P}^{m},
$$

где $m+1$ - число мономиальных слагаемых в $P(z)$, а $B$ - некоторое подмножество коразмерности $m+1$. А именно, в однородных координатах $\left[w_{0}: \ldots: w_{m}\right]$ задается равенство

$$
w_{i}=z_{0}^{\alpha_{0}} \ldots z_{n}^{\alpha_{n}}
$$

где правая часть есть выражение $(i+1)$-го монома в $P(z)$. Это отображение корректно определено вне подмножества $B$, определяемого $m+1$ уравнением: каждый моном приравнивается нулю. Общий слой отображения $\psi$ есть гладкое комплексное подмногообразие в $\mathbb{C P}^{n}-B$ коразмерности $m$, инвариантное относительно некоторого набора символов, выделяемых $m$ линейными условиями на критические значения из множества диагональных в данной системе координат символов. Другими словами, мы получаем ситуацию, когда на $\mathbb{C P}^{n}$ есть набор данных $\left(f_{1}, \ldots, f_{k}, \psi\right)$, где $k=n-m$. Из этого замечания вытекает определение некоторой новой структуры, предлагаемое в следующем разделе.

\section{3. ПСЕВДОТОРИЧЕСКИЕ СТРУКТУРЫ}

Конструкция неторических лагранжевых слоений проективной плоскости, предложенная Ору, может быть обобщена в двух направлениях. Во-первых, можно рассмотреть неторические многообразия, которые обладают символами и инвариантными пучками. Во-вторых, можно от пучков переходить к семействам, следуя рассуждениям, приведенным в конце раздела 2. Мы начнем с примеров. 
Как было показано в работе [3], невырожденная двумерная квадрика обладает инвариантными пучками и может быть расслоена на лагранжевы торы разными способами. Она является торическим мнгообразием Фано, но, если увеличить размерность на единицу и рассмотреть трехмерную невырожденную квадрику $Q$ в $\mathbb{C P}^{4}$, она уже не будет торической. Покажем, что несмотря на это $Q$ может быть расслоена на лагранжевы и изотропные торы (симплектическая структура в дальнейшем всегда предполагается ограниченной с объемлющего проективного пространства).

ПримеР 1. Возьмем квадрику $Q \subset \mathbb{C P}^{4}$, определяемую уравнением

$$
z_{0}^{2}+z_{1} z_{2}+z_{3} z_{4}=0
$$

в системе однородных координат $\left[z_{0}: \ldots: z_{4}\right]$. Выделим подпространство в пространстве отображений моментов (или в $\left.C_{q}^{\infty}\left(\mathbb{C P}^{4}, \mathbb{R}\right)\right)$, состоящее из функций, гамильтоновы векторные поля которых сохраняют $Q$. Пусть диагональный символ $f$ имеет критические значения $\left(\lambda_{0}, \ldots, \lambda_{4}\right)$; он сохраняет нашу квадрику, если и только если

$$
2 \lambda_{0}=\lambda_{1}+\lambda_{2}=\lambda_{3}+\lambda_{4}
$$

Очевидно, что существуют только два таких функционально независимых символа, поэтому $Q$ не является торическим многообразием. Для дальнейших действий нам потребуется пучок дивизоров на $Q$, инвариантных относительно этих двух символов. Такой пучок может быть построен следующим образом: заметим, что последнее условие на $\lambda_{i}$ влечет, что эти символы сохраняют не только нашу квадрику, но целое семейство квадрик. Если мы выберем сохраняющими $Q$ два отображения моментов $f_{1}, f_{2}$, например

$$
f_{1} \mapsto(0,1,-1,2,-2), \quad f_{2} \mapsto(0,2,-2,1,-1),
$$

то те же функции должны сохранять и любую квадрику вида

$$
Q_{w}=\left\{w_{0} z_{0}^{2}+w_{1} z_{1} z_{2}+w_{2} z_{3} z_{4}=0\right\} .
$$

Таким образом, семейством инвариантных квадрик будет $\mathbb{C P}^{2}$ с координатами $\left[w_{0}: w_{1}: w_{2}\right]$. Выберем в этом $\mathbb{C P}^{2}$ прямую, не проходящую через точку $[1: 1: 1]$ и представляющую в общем случае гладкие квадрики (т.е. ее уравнение не может иметь вид $\left.w_{i}=0\right)$. Обозначим эту прямую как $\mathbb{C P}_{u}^{1}$, а квадрики, представляемые ее точками, - как $Q_{u}$. Тогда пересечения $D_{u}=Q \cap Q_{u} \subset Q$ доставляют в общем случае гладкие дивизоры, составляющие инвариантный пучок относительно ограничений $\left.f_{1}\right|_{Q},\left.f_{2}\right|_{Q}$ без базисных компонент. Доказательство этого факта есть простое упражнение из основ алгебраической геометрии. Базисное множество $B$ пучка $\left\{D_{u}\right\}$ задано уравнениями

$$
z_{0}=z_{1} z_{2}=z_{3} z_{4}=0
$$

что представляет собой четыре проективные прямые в $Q$. Пучок содержит три особых элемента, следовательно, отображение

$$
\psi: Q-B \rightarrow \mathbb{C P}_{u}^{1}
$$


выделяет три точки на $\mathbb{C P}_{u}^{1}$, соответствующие этим особым слоям. Если бы особых слоев было два, то наша квадрика была бы торическим многообразием. Поэтому для произвольной морсовской функции $h: \mathbb{C P}_{u}^{1} \rightarrow \mathbb{R}$ индуцируемое слоение обязано иметь особые слои. Исследуем этот пример более тщательно и рассмотрим следующий пучок квадрик $Q_{w}$ :

$$
\mathbb{C P}_{w}^{1}=\left\{w_{0}-w_{1}+w_{2}=0\right\}
$$

Тогда следующие три квадрики дают особые дизизоры $D_{u}$ после пересечения с $Q$ :

$$
Q_{1}=\left\{z_{1} z_{2}+z_{3} z_{4}=0\right\}, \quad Q_{2}=\left\{z_{0}^{2}+z_{1} z_{2}=0\right\}, \quad Q_{3}=\left\{z_{0}^{2}-z_{3} z_{4}=0\right\}
$$

Соответствующими дивизорами являются $D_{1}$ - двойная двумерная квадрика (с гладким носителем); $D_{2}$ - два конуса над одной и той же коникой $q_{1}=\left\{z_{0}^{2}+z_{1} z_{2}=0\right.$, $\left.z_{3}=z_{4}=0\right\}$, являющейся особым множеством этого слоя; $D_{3}$ - два конуса над одной и той же коникой $q_{2}=\left\{z_{0}^{2}-z_{3} z_{4}=0, z_{1}=0, z_{2}=0\right\}$, являющейся особым множеством этого слоя. Симплекс вырождения пары $\left.f_{1}\right|_{Q},\left.f_{2}\right|_{Q}$ состоит из четырех прямых, составляющих базисное множество пучка, и двух коник $q_{1}, q_{2}$.

Рассмотрим теперь морсовскую функцию $h$ на $\mathbb{C P}_{u}^{1}$, определяемую уравнением

$$
h=\frac{\left|w_{1}-w_{2}\right|^{2}-\left|w_{1}\right|^{2}}{\left|w_{0}-w_{1}+w_{2}\right|^{2}+\left|w_{1}\right|^{2}+\left|w_{1}-w_{2}\right|^{2}}
$$

на прямой $\mathbb{C P}_{u}^{1}$. Она имеет две критические точки: максимум в $p_{1}=[0: 1: 1]$ и минимум в $p_{2}=[1: 0:-1]$, соответствующие особым слоям $D_{1}$ и $D_{3}$. Такой выбор $h$ определяет лагранжево слоение $Q-\left(D_{1} \cup D_{3}\right)$. Существует еще один особый слой $D_{2}$, проектирующийся в точку на линии уровня $\gamma_{0}=\{h=0\}$, поэтому получаемое слоение включает в себя одномерное подсемейство особых торов, моделируемых трехмерным тором со стянутым в петлю двумерным подтором. Эти петли лежат на конике $q_{1}$ и расслаивают ее вне двух точек, получаемых пересечением с базисным множеством.

Отступление: монодромия. Здесь уместно упомянуть важное свойство нашей конструкции - возможность простого вычисления монодромии получаемого лагранжева слоения вокруг особых слоев. Монодромия является препятствием к существованию глобальных переменных действие-угол, т.е. она есть мера того, насколько наша система далека от вполне интегрируемой в классическом смысле.

Выделим открытую часть в $Q-B$ условием, что эта часть есть прообраз при отображении

$$
\psi: Q-B \rightarrow \mathbb{C P}_{u}^{1}
$$

кольца

$$
A_{\delta}=\{-\delta<h<\delta\} \subset \mathbb{C P}_{u}^{1},
$$

где $0<\delta<1$ - вещественный параметр. Тогда лагранжево слоение на $Q$, заданное тройкой $\left(\left.f_{1}\right|_{Q},\left.f_{2}\right|_{Q}, \psi^{*} h\right)$ коммутирующих функций, может быть спроектировано 
"отображением действия"

$$
F_{\text {act }}=\left(\left.f_{1}\right|_{Q}, f_{2} \mid Q, \psi^{*} h\right): \psi^{-1}\left(A_{\delta}\right) \rightarrow \mathbb{R}^{3}=\mathbb{R}\langle x, y, z\rangle,
$$

и имеется одномерное множество $I$ в $\operatorname{Im} F_{\text {act }} \subset \mathbb{R}\langle x, y, z\rangle$, определяемое в координатах как отрезок

$$
I=\{2 x=y, z=0,-1<x<1\} .
$$

Таким образом, трехмерное пространство лагранжевых слоев $\operatorname{Im} F_{\text {act }}$ содержит одномерное семейство особых лагранжевых торов. Рассмотрим петлю $\gamma \subset \operatorname{Im} F_{\text {act }}$, обходящую вокруг этого особого отрезка. Возникает естественный вопрос - чему равна монодромия вдоль этой петли как элемент End $H_{1}\left(T^{3}, \mathbb{Z}\right)$ ? Ответ содержит следующее

ПРЕДЛОЖЕНИЕ 3. Оператор монодромии $\operatorname{Mon}_{\gamma} \in$ End $H^{1}\left(T^{3}, \mathbb{Z}\right)$ выражается в терминах "исчезающих ииклов" особого слоя $D_{2}^{0}=D_{2}-B$.

ДокАЗАТЕльство. Для общего гладкого слоя в $Q-B$, являющегося гладкой двумерной квадрикой без четырех прямых, гомеоморфной $C^{*} \times C^{*}$, группа гомологий представлена прямой суммой $\mathbb{Z}\left[h_{1}\right] \oplus \mathbb{Z}\left[h_{2}\right] ;$ для особого слоя $D_{2}$ без пересечения с теми же прямыми группа редуцируется до $\mathbb{Z}[h]$, как у одномерной квадрики без двух точек. Таким образом, наш “исчезающий цикл" есть образующая в $H_{1}\left(D_{u}^{0}, \mathbb{Z}\right)$, исчезающая при переходе к особому слою. В нашем случае оператор монодромии $\mathrm{Mon}_{\mathrm{van}}$ принадлежит End $H_{1}\left(D_{u}^{0}, \mathbb{Z}\right)$, при этом, поскольку имеется вложение

$$
H_{1}\left(D_{u}^{0}, \mathbb{Z}\right) \subset H_{1}\left(T^{3}, \mathbb{Z}\right),
$$

где $T^{3}$ - гладкий слой $\operatorname{Im} F_{\text {act }}$, искомая монодромия $\mathrm{Mon}_{\gamma}$ есть просто прямая сумма:

$$
\operatorname{Mon}_{\gamma}=\operatorname{Id}_{1} \oplus \operatorname{Mon}_{\mathrm{van}} .
$$

Заметим, что подобные монодромные соотношения всегда имеют место для лагранжевых слоений, задаваемых инвариантными голоморфными пучками.

Обобщим теперь последний пример и рассмотрим случай, когда число символов, сохраняющих $n$-мерное многообразие, меньше $n-1$.

Пример 2. Возьмем гладкую невырожденную пятимерную квадрику $Q \subset \mathbb{C P}^{6}$ с уравнением

$$
Q=\left\{z_{0}^{2}+z_{1} z_{2}+z_{3} z_{4}+z_{5} z_{6}=0\right\} .
$$

Если гамильтоново действие какого-то символа сохраняет эту квадрику, то его критические значения удовлетворяют условиям

$$
2 \lambda_{0}=\lambda_{1}+\lambda_{2}=\lambda_{3}+\lambda_{4}=\lambda_{5}+\lambda_{6},
$$

и существуют в точности три функционально независимых символа, подходящих под эти условия. Обозначим их через $f_{1}, f_{2}, f_{3}$. Поэтому для того чтобы наша конструкция была применима, нам нужно найти некоторое двумерное торическое многообразие $Y$ вместе с отображением $\psi$ из $Q$ в $Y$. 
Рассмотрим отображение из $\mathbb{C P}^{6}$ с координатами $\left[z_{0}: \ldots: z_{6}\right]$ в $\mathbb{C P}^{3}$ с координатами $\left[w_{0}: \ldots: w_{3}\right]$, задаваемое уравнениями

$$
w_{0}=z_{0} z_{1}, \quad w_{1}=z_{2} z_{3}, \quad w_{2}=z_{3} z_{4}, \quad w_{3}=z_{5} z_{6}
$$

(именно о таком отображении мы говорили в конце раздела 2). Это отображение корректно определено вне набора проективных двумерных плоскостей, когда все $w_{i}=0$. Это множество состоит из $2^{4}=16$ таких плоскостей. Обозначим объединение их через $B_{0}$. Тогда имеется комплексное отображение

$$
\psi_{0}: \mathbb{C P}^{6}-B \rightarrow \mathbb{C P}_{w}^{3}
$$

Рассмотрим ограничение этого отображения на квадрику $Q$ без базисного множества $B=Q \cap B_{0}:$

$$
\psi: Q-B \rightarrow \mathbb{C P}_{w}^{2},
$$

так как образ квадратики $Q$ в $\mathbb{C P}_{w}^{3}$ есть в точности проективная плоскость $\mathbb{C P}^{2}$, определяемая уравнением

$$
w_{0}+w_{1}+w_{2}+w_{3}=0 .
$$

Слои $\psi$ комплексны, общий слой гладкий, и ключевым является тот факт, что гамильтоново действие каждого $f_{i}$ сохраняет каждый слой. Особые слои лежат над четырьмя проективными прямыми $l_{j}$, выделенными условиями

$$
\sum_{i=0}^{3} w_{i}=0, \quad w_{j}=0 .
$$

Ели бы таких прямых было только три, то существовала бы торическая структура на нашем $Q$. В нашем случае мы можем взять на $\mathbb{C P}_{w}^{2}$ два коммутирующих отображения моментов (символов) $h_{1}$ и $h_{2}$ с множеством вырождения $\Delta_{h}=l_{1} \cup l_{2} \cup l_{3}$, и тогда справедливо следующее

ПреДЛОЖЕНИЕ 4. Въбор таких символов $h_{1}$ u $h_{2}$ на $\mathbb{C P}_{w}^{2}$ задает слоение на $Q$, общим слоем которого является гладкий лагранжев тор.

ДокАзАтЕльство. Для открытой части $Q-B_{0}$ имеется набор функций $\left(f_{1}, f_{2}, f_{3}\right.$, $\left.\psi^{*} h_{1}, \psi^{*} h_{2}\right)$, которые коммутируют между собой. Коммутационное соотношение $\left\{f_{i}, f_{j}\right\}_{\omega}=0$ очевидно; коммутационные соотношения для $f_{i}$ и $\psi^{*} h_{j}$ следуют из тех же рассуждений, что и в теореме 1 из работы [3], наконец, коммутационные соотношения для $\psi^{*} h_{1}$ и $\psi^{*} h_{2}$ следуют из того, что соответствующий тор $T^{2} \subset \mathrm{Sym} \mathbb{C P}_{w}^{2}$ вкладывается в $\operatorname{Sym}(Q-B)$, и это вложение является гомоморфизмом групп. Таким образом, почти всё $Q$ несет полный набор первых интегралов, что вообще-то еще не влечет компактность общего слоя. Но в нашей ситуации общий слой можно построить "руками" и проверить его компактность. Выберем сначала клиффордов тор

$$
T_{\left(c_{4}, c_{5}\right)}^{2} \subset \mathbb{C P}_{w}^{2}
$$


задаваемый парой регулярных значений $h_{1}$ и $h_{2}$, так, чтобы

$$
T_{\left(c_{4}, c_{5}\right)}^{2} \cap l_{0}=\varnothing
$$

(заметим, что это верно для общих регулярных значений). Зафиксируем точку $p \in T_{\left(c_{4}, c_{5}\right)}^{2} \subset \mathbb{C P}_{w}^{2}$ и рассмотрим слой

$$
N^{0}(p)=\psi^{-1}(p) \subset Q-B
$$

открытую часть гладкого комплексного подмногообразия $N(p) \subset Q$, которое уже является торическим с интегралами $\left.f_{i}\right|_{N(p)}$. Возможность компактификации очевидна: замыкание дается пересечением трех невырожденных квадрик в $\mathbb{C P}^{6}$. Выберем теперь некоторые регулярные значения для $f_{1}, f_{2}, f_{3}$ и рассмотрим совместное множество уровня

$$
T_{(p)}^{3}=\left\{\left.f_{i}\right|_{N(p)}=c_{i}, i=1,2,3\right\} \subset N(p) .
$$

Заметим, что для общих значений $c_{i}$ это подмножество содержится в $N^{0}(p)$.

Пусть теперь $p$ варьируется в $T_{\left(c_{4}, c_{5}\right)}^{2} \subset \mathbb{C P}_{w}^{2}$. Объединение

$$
T_{\left(c_{1}, \ldots, c_{5}\right)}^{5}=\bigcup_{p \subset T_{\left(c_{4}, c_{5}\right)}^{2}} T_{(p)}^{3}
$$

собирается в гладкий компактный пятимерный тор. Этот тор является лагранжевым, так как его касательное пространство $T_{s} T$ в каждой точке натянуто на гамильтоновы векторные поля $X_{f_{i}}, X_{\psi^{*} h_{j}}$ коммутирующих функций. Этим завершается доказательство.

ЗАмечАниЕ 2. Конечно, выше можно было взять любую пару коммутирующих отображений моментов на $\mathbb{C P}_{w}^{2}$ и получить другое слоение, общим слоем которого снова будет гладкий лагранжев тор, но множество особых слоев при этом будет гораздо больше. Мы взяли именно такие функции $h_{1}, h_{2}$ для того, чтобы получить минимальное по числу особенностей лагранжево слоение.

ЗАмечАниЕ 3. Условие, что тор $T^{n-k}$ с подлежащего многообразия $Y$ поднимается посредством $\psi^{*}$ до коммутативной подгруппы в $\operatorname{Sym}(Q-B)$, используемое нами для установления коммутационного соотношения для поднятых функций, является очень сильным. Так как наша цель - делать все в как можно более широких предположениях, ниже в базовом определении мы используем более слабое условие, подходящее и для символов, а не только для отображений моментов.

Заметим также, что в нашей ситуации мы можем применять рассуждения, аналогичные приведенным выше, для получения, во-первых, связи свойства несдвигаемости с условием Бора-Зоммерфельда относительно антиканонического класса; во-вторых, выражения для монодромии в терминах исчезающих образующих в гомологиях слоев.

Формализуем наше обсуждение, введя следующее определение. 
ОПРЕДЕЛЕНИЕ 1 . Пусть $\left(X, \omega_{X}\right)$ - симплектическое многообразие вещественной размерности $2 n$ с симплектической формой $\omega_{X}$. Пусть набор данных $\left(f_{1}, \ldots, f_{k}, B\right.$, $\left.\psi,\left(Y, \omega_{Y}\right)\right)$ состоит из:

1) $k$ гладких вещественных функционально независимых почти всюду морсовских функций $f_{i}$, коммутирующих между собой в смысле скобки Пуассона;

2 ) симплектического подмногообразия $B \subset X$ вещественной размерности $2(k-1)$;

$3)$ семейства симплектических подмногообразий вещественной размерности $2 k$ с базисным множеством $B$, общий элемент которого гладкий, покрывающее всё $X$ и параметризуемое торическим симплектическим многообразием $Y$ вещественной размерности $2(n-k)$;

4) отображения $\psi: X-B \rightarrow Y$, определяемого данным семейством подмногообразий таким образом, что произвольный элемент семейства, соответствующий точке $p \in Y$, представляется в виде

$$
D_{p}=\overline{\psi^{-1}(p)}=\psi^{-1}(p) \cup B ;
$$

причем выполнены следующие условия согласованности: для любой точки $p \in Y$ и любого $i=1, \ldots, k$ гамильтоново векторное поле $X_{f_{i}}$ параллельно элементу семейства $D_{p}$ в каждой его точке, и для любой гладкой функции $h$ на $Y$ имеет место соотношение

$$
X_{\psi^{*} h}=\tau \nabla_{\psi} X_{h}
$$

на $X-(B \cup \operatorname{Sing})$, где $\nabla_{\psi}$ - симплектическая связность, индуцируемая отображением $\psi, \tau$ - некоторая положительная функция, $X_{\psi^{*} h}$ и $X_{f}$ - гамильтоновы векторные поля, определяемые относительно симплектических форм $\omega_{X}$ и $\omega_{Y}$ соответственно, a Sing - множество особых точек особых слоев отображения $\psi$.

Тогда мы будем называть такой набор $\left(f_{1}, \ldots, f_{k}, B, \psi,\left(Y, \omega_{Y}\right)\right)$ (или, кратко, $\left.\left(f_{1}, \ldots, f_{k}, \psi\right)\right)$ псевдоторической структурой на $X$.

ЗАмечАниЕ 4. Нетрудно видеть, что условие (9) выполнено автоматически, если отображение $\psi$ комплексно и комплексная размерность $Y$ равна 1. Поэтому в случае инвариантных пучков это условие пропадает.

Можно сопоставить псевдоторической структуре некоторые целочисленные инварианты. Простейшим является следующий.

ОПРЕДЕЛЕНИЕ 2. Комплексная размерность $Y$ называется рангом nсевдоторической структуры.

Таким образом, в примерах 1 и 2 мы встречались со псевдоторическими структурами рангов 1 и 2.

Имеется следующее обобщение предложения 3.

Теорема 1. Пусть $X$ - псевдоторическое многообразие Фано с фиксированной псевдоторической структурой $\left(f_{1}, \ldots, f_{k}, \psi\right)$. Тогда произвольный выбор множества алгебраччески независимых морсовских функций $h_{j} \in C^{\infty}(Y, \mathbb{R})$ в инволючии индуцирует изотропное слоение на $X$, общим слоем которого является гладкий лагранжев тор. 
ДокАзАТЕЛЬство повторяет рассуждения из теоремы 1 в работе [3] и предложения 3. Пусть $\left(h_{1}, \ldots, h_{n-k}\right)$ - набор коммутирующих алгебраически независимых морсовских функций на $Y$. Для симплектического отображения $\psi$ рассмотрим подъемы $\psi^{*} h_{j}$, определяющие функции на $X-B$, гладкие на $X-(B \cup \operatorname{Sing})$ где $B$ - базисное множество и Sing - множество особенностей слоев. Однако мы предположили, что общий слой гладкий, поэтому множество особых слоев составляет подмножество в $Y$ размерности меньше чем $n-k$. Таким образом, дополнение $X-(B \cup \operatorname{Sing})$ несет на себе набор $\left(f_{1}, \ldots, f_{k}, \psi^{*} h_{1}, \ldots, \psi_{n-k}^{*} h_{n-k}\right)$ гладких функций. Более того, нетрудно видеть, что эти функции коммутируют. В самом деле, $f_{i}$ и $f_{j}$ коммутируют по определению псевдоторической структуры. Гамильтоновы векторные поля $X_{\psi^{*} h_{j}}$ симплектически ортогональны слоям $\psi$, в то время как гамильтоновы векторные поля $X_{f_{i}}$ параллельны им, что влечет

$$
\left\{f_{i}, \psi^{*} h_{j}\right\}=\omega_{X}\left(X_{f_{i}}, X_{\psi^{*} h_{j}}\right)=0
$$

для всех $i$ и $j$. Рассмотрим теперь скобки

$$
\left\{\psi^{*} h_{i}, \psi^{*} h_{j}\right\}=d\left(\psi^{*} h_{i}\right)\left(X_{\psi^{*} h_{j}}\right) .
$$

Опираясь на условие (9), равенство можно продолжить:

$$
\left\{\psi^{*} h_{i}, \psi^{*} h_{j}\right\}=d\left(\psi^{*} h_{i}\right)\left(\tau \nabla_{\psi} X_{h_{j}}\right)=\tau \psi^{*}\left(d h_{i}\left(X_{h_{j}}\right)\right)=0
$$

на $X-(B \cup \operatorname{Sing})$.

Заметим, что гамильтоновы векторные поля $X_{f_{1}}, \ldots, X_{f_{k}}, X_{\psi^{*} h_{1}}, \ldots, X_{\psi^{*} h_{n-k}}$ алгебраически независимы почти всюду; последние в наборе поля вырождаются над симплексом вырождения $\Delta_{h} \subset Y$ набора $\left(h_{1}, \ldots, h_{n-k}\right)$. Соответствующие слои могут быть объединены в многокомпонентный приводимый дивизор

$$
D_{h}=\psi^{-1}\left(\Delta_{h}\right) \subset X .
$$

Если обозначить через $\Delta_{k}$ симплекс вырождения набора $\left(f_{1}, \ldots, f_{k}\right)$,

$$
\Delta_{k}=\left\{X_{f_{1}} \wedge \cdots \wedge X_{f_{k}}=0\right\}
$$

то дополнение $X-\left(B \cup D_{h} \cup \Delta_{k}\right)$ наделяется вещественной поляризацией, натянутой на наши гамильтоновы векторные поля. Однако необходимы дополнительные аргументы, так как далеко не всякое лагранжево распределение интегрируемо.

Первое наблюдение касается базисного множества $B$ отображения $\psi$, слои которого сохраняются функциями $f_{1}, \ldots, f_{k}$. Условие согласованности влечет инвариантность базисного множества $B$ при гамильтоновом действии каждой $f_{i}$. Из того же условия следует инвариантность множества особенностей Sing относительно гамильтонова действия каждой $f_{i}$. Рассмотрим "отображение действия"

$$
F_{\mathrm{a}}=\left(f_{1}, \ldots, f_{k}\right): X \rightarrow \mathbb{R}^{k}
$$

и обозначим образ $F_{\mathrm{a}}(X)$ как $P_{k}$. Это связная ограниченная $k$-мерная область в $\mathbb{R}^{k}$; образ $B$ обозначим как $F_{\mathrm{a}}(B)$. Инвариантность $B$ относительно каждой $X_{f_{i}}$ 
показывает, что $F_{\mathrm{a}}(B)$ имеет вещественную коразмерность 1 в $P_{k}$. По тем же причинам вещественная коразмерность $F_{\text {a }}($ Sing) меньше или равна 1 . Следовательно, для общей внутренней точки $P_{k}$, представляемой набором некритических значений $\left(c_{1}, \ldots, c_{k}\right)$, совместное множество уровня

$$
S_{\left(c_{1}, \ldots, c_{k}\right)}=\left\{f_{i}=c_{i}\right\}
$$

есть гладкое компактное коизотропное многообразие, не пересекающее ни $B$, ни Sing. В то же время, так как граница $\partial P_{k}$ есть образ $\Delta_{k}$, каждое $X_{f_{i}}$ не обращается в нуль на $S_{\left(c_{1}, \ldots, c_{k}\right)}$.

Далее, так как $S_{\left(c_{1}, \ldots, c_{k}\right)}$ не имеет общих точек с $B$ и Sing, функции $f_{k+1}, \ldots, f_{n}$ такие, что

$$
f_{k+j}=\left.\psi^{*} h_{j}\right|_{S_{\left(c_{1}, \ldots, c_{k}\right)}},
$$

корректно определены и гладкие; коммутационные соотношения для этих ограничений остаются прежними. Выберем теперь набор некритических значений $\left(c_{k+1}, \ldots, c_{n}\right)$ функций $h_{1}, \ldots, h_{n-k}$, обладающий следующим свойством: соответствующий тор Клиффорда $T_{\left(c_{k+1}, \ldots, c_{n}\right)}^{n-k}$ гладкий и не пересекает $\psi(\operatorname{Sing}) \subset Y$ (очевидно, что это также общий выбор). Рассмотрим совместное множество уровня

$$
S_{\left(c_{1}, \ldots, c_{k}\right)} \supset T_{\left(c_{1}, \ldots, c_{k}, c_{k+1}, \ldots, c_{n}\right)}=\left\{f_{k+i}=c_{k+i}, i=1, \ldots, n-k\right\} .
$$

Это множество является гладким компактным подмногообразием в $X$ вещественной размерности $n$. Гладкость следует из того, что некритические множества уровня коммутирующих алгебраически независимых морсовских функций пересекаются трансверсально. С другой стороны, имеются $n$ линейно независимых в каждой точке гамильтоновых векторных полей, параллельных $T_{\left(c_{1}, \ldots, c_{n}\right)}$, откуда следует, что $S_{\left(c_{1}, \ldots, c_{k}\right)}$ - это гладкий лагранжев тор.

Что остается в $X$ не охваченным такими гладкими лагранжевыми торами? Множество коммутирующих функций $\left(h_{1}, \ldots, h_{n-k}\right)$ обладает своим собственным множеством вырождения $\Delta_{h} \subset Y$, состоящим из симплектических дивизоров $D_{1}, \ldots, D_{m} \subset Y$; подмногообразие $\Delta_{h}$ расслоено на изотропные торы размерности меньше $n-k$. Таким образом, необходимо вырезать $\overline{\psi^{-1}\left(\Delta_{h}\right)}$ из нашего псевдоторического многообразия $X$, чтобы получить лагранжево слоение. Особенности лагранжевых слоев возникают из-за особенностей слоев: пусть

$$
Y \supset \operatorname{Sing}_{Y}=\psi(\operatorname{Sing})
$$

тогда для гладкого клиффордова тора $T_{\left(c_{k+1}, \ldots, c_{n}\right)}^{n-k} \subset Y$, пересекающего $\operatorname{Sing}_{Y}$, cyществуют значения $c_{1}, \ldots, c_{k}$ функций $f_{1}, \ldots, f_{k}$ такие, что совместное множество уровня $T_{\left(c_{1}, \ldots, c_{k}, c_{k+1}, \ldots, c_{n}\right)}$ есть особый лагранжев тор. Особенности возникают, если набор $\left(X_{f_{1}}, \ldots, X_{f_{k}}\right)$ вырождается, это происходит в некоторых подмножествах $\Delta_{k} \subset X$.

Дивизор

$$
D_{\psi, h}=\overline{\psi^{-1}\left(\Delta_{h}\right)} \subset X
$$


очень важен в наших рассмотрениях; мы будем называть псевдоторическую структуру канонической, если для некоторого набора интегралов $\left(h_{1}, \ldots, h_{n-k}\right)$ дивизор $D_{\psi, h}$ представляет класс гомологий, двойственный по Пуанкаре антиканоническому классу $-K_{X} \in H^{2}(X, \mathbb{Z})$. Заметим, что в некоторых случаях (скажем, в примере Ору) двойственный по Пуанкаре класс $\left[D_{\psi, h}\right]$ может быть разным в зависимости от набора $\left(h_{1}, \ldots, h_{n-k}\right)$.

Назовем гладкий лагранжев тор $T \subset X$ тором Opy если он получается с помощью представленной выше конструкции из набора согласованных данных $\left(f_{1}, \ldots, f_{k}, \psi\right)$ для некоторых коммутирующих морсовских функций $h_{1}, \ldots, h_{n-k}$ на многообразии $Y$.

Естественно расширить обсуждение свойства несдвигаемости и на этот случай

ГиПотезА. Тор Ору $T \subset X$ в псевдоторическом многообразии Фано несдвигаем, если только он бор-зоммерфелъдов относительно антиканонического класса.

Но вернемся к нашим примерам. Примеры 1 и 2 могут быть легко обобщены в виде следующего утверждения.

Теорема 2. Произвольная гладкая квадрика $Q$ обладает псевдоторической структурой.

ДокАЗАТЕЛЬСтво. Пример пятимерной квадрики в $\mathbb{C P}^{6}$ подробно разобран выше, и доказательство теоремы просто следует этому образцу. В зависимости от четности $n$ уравнением рассматриваемой квадрики будет или

$$
z_{0}^{2}+z_{1} z_{2}+\cdots+z_{n-1} z_{n}=0
$$

если $n$ четно, или

$$
z_{0} z_{1}+\cdots+z_{n-1} z_{n}=0
$$

если $n$ нечетно. В первом случае пространство символов, сохраняющих $Q$, имеет размерность $n / 2$; во втором - размерность $(n+1) / 2$. Положив

$$
w_{0}=z_{0}^{2}, \quad w_{1}=z_{1} z_{2}, \quad \ldots, \quad w_{n / 2}=z_{n-1} z_{n}
$$

в первом случае и

$$
w_{0}=z_{0} z_{1}, \quad \ldots, \quad w_{(n-1) / 2}=z_{n-1} z_{n}
$$

во втором, мы получаем отображение $\psi$ в соответствующее проективное пространство $\mathbb{C P}^{k}$ всего нашего проективного пространства $\mathbb{C P}^{n}$, причем квадрика $Q$ оказывается расслоенной над гиперпространством $\mathbb{C P}^{k-1} \subset \mathbb{C P}^{k}$, определяемым уравнением

$$
w_{0}+\cdots+w_{k}=0 .
$$

Геометрически отображение $\psi$ представляется следующим образом. Рассмотрим в полном линейном ряду $|2 H|$ квадрик на $\mathbb{C P}^{n}$ проективное подпространство, состоящее из квадрик, удовлетворяющих следующему условию: эти квадрики инвариантны относительно тех же символов, что и $Q$. Нетрудно видеть, что такие квадрики 
составляют некоторое пространство $\mathbb{C P}^{k} \subset|2 H|$. Так как кэлерова структура на $\mathbb{C P}^{n}$ фиксирована в наших построениях, проективное пространство $\mathbb{C P}^{n}$ вкладывается полным линейным рядом сначала в двойственное к $|2 H|$, а затем по двойственности и в само $|2 H|$. Обозначим образ этого вложения через $F_{2}$. Базисное множество $B$, в котором введенное формально выше $\psi$ не определено, представляется пересечением $F_{2}$ с набором проективных пространств коразмерности $k+1$, который составляет подмногообразие $B_{1} \subset|2 H|$. Нетрудно видеть, что отображение $\psi$ геометрически представляется просто как проекция $F_{2}$ из $B_{1}$ в $\mathbb{C P}^{k}$. Так как обе симплектические формы на $F_{2}$ и $\mathbb{C P}^{k}$ получаются ограничением стандартной формы с $|2 H|$, условие $(9)$ для $F_{2}$ и $\mathbb{C P}^{k}$ выполяется для любой гладкой функции на $\mathbb{C P}^{k}$. Заметим, что симплектические формы на $F_{2}$ и $\mathbb{C P}^{n}$ отличаются на постоянный множитель, поэтому условие (9) выполняется и для симплектических форм на $\mathbb{C P}^{n}$ и $\mathbb{C P}^{k}$. Остается заметить, что условие (9) справедливо для ограничений на подмногообразия, каковыми могут быть выбраны $Q$ и $\mathbb{C P}^{k-1}$. Отсюда получаем псевдоторическую структуру на $Q$. Теорема доказана.

Из этой теоремы вытекает существование псевдоторических структур на некоторых полных пересечениях квадрик в $\mathbb{C P}^{n}$. В самом деле, прообразом произвольного проективного подпространства $\mathbb{C P}^{l} \subset \mathbb{C P}^{k}$ при отображении $\psi$ будет пересечение $k-l$ квадрик. Однако эти квадрики не общие, поскольку все они имееют одни и те же сохраняющие их символы. Например, гладкая поверхность дель Пеццо степени 4 не обладает псевдоторической структурой ранга 1 , хотя и является пересечением двух квадрик в $\mathbb{C P}^{4}$.

Далее, возникает естественный вопрос: какие многообразия Фано допускают псевдоторические структуры?

Нетрудно видеть, что любое торическое многообразие является автоматически псевдоторическим. Случай невырожденных квадрик, являющийся предметом теоремы 2, может быть расширен за счет полных пересечений гиперплоскостей одинаковой степени и т.п. С другой стороны, интересен и такой вопрос: сколъко разных псевдоторических структур может допускать многообразие Фано?

Пример 3. Рассмотрим четырехмерную невырожденную квадрику $Q \subset \mathbb{C P}^{5}$ и выберем однородные координаты $\left[z_{0}: \ldots: z_{5}\right]$ так, чтобы $Q$ имела уравнение

$$
z_{0}^{2}+z_{1}^{2}+z_{2}^{2}+z_{3}^{2}+z_{4} z_{5}=0
$$

Тогда только один диагональный символ $f$ будет сохранять $Q$,

$$
f=\frac{\left|z_{4}\right|^{2}-\left|z_{5}\right|^{2}}{|z|^{2}}
$$

причем этот символ будет вырожденным - он имеет одномерное критическое подмножество в $Q$. Рассмотрим отображение

$$
\psi: Q \rightarrow \mathbb{C P}^{3},
$$


являющееся ограничением отображения

$$
\psi_{0}: \mathbb{C P}^{5} \rightarrow \mathbb{C P}^{4}
$$

которое задается как

$$
w_{0}=z_{0}^{2}, \quad w_{1}=z_{1}^{2}, \quad w_{2}=z_{2}^{2}, \quad w_{3}=z_{3}^{2}, \quad w_{4}=z_{4} z_{5} .
$$

Продолжая движение в этом направлении, мы получаем псевдоторическую структуру ранга 3 , в то время как теорема 2 предлагает универсальную псевдоторическую структуру ранга 2 .

\section{4. ЗАКЛЮЧИТЕЛЬНЫЕ ЗАМЕЧАНИЯ}

Напомним, что исходным пунктом для настоящей работы послужил пример Ору, в котором проективная плоскость расслаивается на специальные лагранжевы торы [8]. Условие специальности для лагранжевых торов знаменито своей ролью в зеркальной симметрии (см., например, работу [12], однако там оно возникает для лагранжевых торов в многообразиях Калаби-Яу). Ору предложил подход, обобщающий понятие специального лагранжева тора на случай многообразий Фано (или, более точно, для открытых многообразий Калаби-Яу). Если на многообразии Фано $X$ зафиксировать некоторый элемент антиканонической системы $D \subset\left|-K_{X}\right|$, то дополнение $X-D$ снабжено старшей голоморфной формой $\theta_{D}$, определенной однозначно с точностью до умножения на константу. Эта форма продолжается до мероморфной старшей формы с полюсом на $D$. Тогда если дополнение $X-D$ расслоено на лагранжевы торы, то на слои может быть наложено условие специальности относительно $\theta_{D}$. Главная гипотеза, высказанная в работе [8], гласит, что для произвольного многообразия Фано $X$ и любого такого $D$ существует слоение на специальные лагранжевы торы (с возможными особыми слоями, конечно). Поэтому главный наш интерес в псевдоторической геометрии представляют примеры псевдоторических лагранжевых слоений многообразий Фано без элемента антиканонической системы.

Если многообразие Фано $X$ обладает псевдоторической структурой $\left(f_{1}, \ldots, f_{k}, \psi\right)$, то возникает вопрос, возможно ли реализовать специальное лагранжево слоение путем выбора некоторых подходящих $h_{j}$. Первый пример был приведен в работе [8], здесь мы предлагаем следующий

ПримеР 4. Рассмотрим невырожденную четырехмерную квадрику $Q \subset \mathbb{C P}^{5}$ и систему однородных координат $\left[z_{0}: \ldots: z_{5}\right]$, в которой $Q$ задана уравнением

$$
z_{0} z_{1}+z_{2} z_{3}+z_{4} z_{5}=0
$$

Возьмем три невырожденных символа (отображения моментов), сохраняющих квадрику, как в теореме 2 , и обозначим их $f_{1}, f_{2}, f_{3}$. Симплекс вырождения $\Delta_{3} \subset Q$ состоит из восьми двумерных плоскостей, лежащих на $Q$, и трех двумерных квадрик. Мы различаем эти две компоненты $\Delta_{3}$, обозначая через $B$ объединение плоскостей 
и через Sing объединение этих трех квадрик. Вырезая $B$ из $Q$, рассматриваем комплексное отображение

$$
\psi: Q-B \rightarrow \mathbb{C P}_{u}^{1},
$$

где $\mathbb{C P}_{u}^{1}$ - проективная прямая в $\mathbb{C P}_{w}^{2}$ с однородными координатами $\left[w_{0}: w_{1}: w_{2}\right]$, определяемая уравнением

$$
\mathbb{C P}_{u}^{1}=\left\{w_{0}+w_{1}+w_{2}=0\right\} \subset \mathbb{C P}_{w}^{2}
$$

Как и выше, отображение $\psi$ задано соотношениями

$$
w_{0}=z_{0} z_{1}, \quad w_{1}=z_{2} z_{3}, \quad w_{2}=z_{4} z_{5}
$$

и не определено в точности на $B$. Нетрудно видеть, что слои $\psi$ инвариантны относительно гамильтонова действия каждой $f_{i}$ и каждый слой компактифицируется до множества нулей некоторого сечения линейного расслоения $2 H$ над $Q$. Восемь проективных плоскостей составляют базисное множество соответствующего пучка инвариантных дивизоров

$$
\left\{D_{u}=\overline{\psi^{-1}(u)}\right\}
$$

(отсюда обозначение $B$ ). Этот пучок имеет в точности три выделенных элемента $\psi^{-1}([0: 1:-1]), \psi^{-1}([1: 0:-1]), \psi^{-1}([-1: 1: 0])$, каждый из которых представляется парой конусов над невырожденной двумерной квадрикой, являющейся множеством особенностей этого элемента (отсюда обозначение этой части $\Delta_{3}$ как Sing). Гладкие слои $\psi$ имеют когомологии $H_{1}\left(D_{u}^{0}, \mathbb{Z}\right)$ ранга 3 , и переход к особому слою убивает одну из образующих в $H_{1}\left(D_{u}^{0}, \mathbb{Z}\right)$, которая есть соответствующий исчезающий генератор особого слоя.

Далее, выбор произвольного символа (отображения моментов) $h$ на $\mathbb{C P}_{u}^{1}$ индуцирует специальное лагранжево слоение на $Q$. Чтобы увидеть это, сначала заметим, что символ $h$ всегда имеет в точности две критические точки $p_{\max }, p_{\min }$ на $\mathbb{C P}_{u}^{1}$, и эти две критические точки выделяют два элемента $D_{\max }, D_{\min }$ в нашем пучке $\left\{D_{u}\right\}$. Поэтому согласно теореме 1 выбор $h$ определяет лагранжево слоение на $Q-\left(D_{\max } \cup D_{\min }\right)$. Объединение $D=D_{\max } \cup D_{\min }$ есть приводимый дивизор из антиканонической системы $\left|-K_{Q}\right|$. В самом деле, по формуле присоединения имеем $-K_{Q}=4 H$, и каждый $D_{*}$ представляет сечение $2 H$.

Для доказательства специальности слоения рассмотрим соответствующую голоморфную форму $\theta_{D}$ и заметим, что она инвариантна относительно отображений моментов $f_{1}, f_{2}, f_{3}$ (так как дивизор $D$ переходит в себя). В то же время имеется семейство определяемых вращениями голоморфных автоморфизмов $Q-B$, которые индуцируются нашей $h$, при этом $\theta_{D}$ инвариантна также и относительно этих автоморфизмов. Поэтому ограничение формы $\theta_{D}$ на любой тор из слоения есть некоторая константа, умноженная на форму объема. Но модуль этой константы различен для различных линий уровня функции $h$ на $\mathbb{C P}_{u}^{1}$, поскольку метрика Фубини-Штуди на $\mathbb{C P}_{u}^{1}$ и кэлерова метрика на $Q-B$ связаны умножением на норму гамильтонова векторного поля $X_{h}$ на $\mathbb{C P}_{u}^{1}$. Однако фазовый множитель будет одним и тем же для всех гладких лагранжевых торов, что и означает специальность нашего слоения. 
Введение псевдоторической структуры позволяет обобщить многие подходы к проблемам и гипотезам, принятые в случае торических многообразий Фано.

В геометрическом квантовании многообразие Фано, обладающее псевдоторической структурой, представляется некоторым общим псевдоторическим слоением, на слои которого, как обычно, налагается условие Бора-Зоммерфельда относительно подходящей поляризации (например, относительно антиканонического расслоения и его степеней). Из этого проистекает сложная и интересная проблема сравнения числа бор-зоммерфельдовых слоев для разных псевдоторических структур.

Для программы зеркальной симметрии, примененной к неторическому многообразию Фано, обладающему псевдоторической структурой, можно обобщить стандартный подход, рассматривая те слои в псевдоторическом слоении, которые имеют нетривиальные когомологии Флоера с собой, и затем надстраивая $A_{\infty}$-категорию на эти выделенные слои.

Для теории интегрируемых систем псевдоторические структуры могут помочь в решении реальных динамических систем на однородных пространствах, грассманианах или некоторых сферических многообразиях.

Нам также кажется очень интересным следующее замечание (последнее в этой работе). Имея некоторую псевдоторическую структуру $\left(f_{1}, \ldots, f_{k}, \psi\right)$ на многообразии $X$, можно определить некоторые классы когомологий $c_{i} \in H^{2 i}(X, \mathbb{Z})$, получающиеся путем подъема посредством $\psi$ и подходящего продолжения на компактификацию слоев касательного расслоения $T Y$. Для псевдоторической структуры ранга 1 это дает единственный класс в $H^{2}(X, \mathbb{Z})$, двойственный по Пуанкаре классу гомологий слоя $D_{u}$. Для структуры ранга $n-k$ эти данные могут быть собраны в вектор

$$
\left(r, c_{1}, \ldots, c_{r}\right) \in H^{2 *}(X, \mathbb{Z}),
$$

где $r$ - ранг этой псевдоторической структуры. Такой вектор может реализоваться разными псевдоторическими слоениями с фиксированными топологическими данными, на множестве которых можно ввести отношение эквивалентности с помощью гамильтоновой изотопии. Тогда пространство всех возможных слоений факторизуется по этому соотношению, и мы получаем некоторые многообразия модулей.

Откуда такая идея? Некоторое время назад один из подходов к зеркальной симметрии предполагал двойственность между векторными расслоениями и лагранжевыми циклами. Но с точки зрения геометрического квантования векторное расслоение не сравнимо с лагранжевым циклом, поскольку квантовое состояние представляется или сечением (с точностью до умножения на константу) векторного расслоения, или лагранжевым циклом. Поэтому векторное расслоение может быть сравнимо с лагранжевым слоением. И, как мы видели выше, некоторые лагранжевы слоения, а именно псевдоторические, характеризуются топологическими данными, очень знакомыми по теории многообразий модулей векторных расслоений.

Последние аргументы пока что выглядят слишком спекулятивно, но мы надеемся продолжить работу и над этой темой. 
Благодарности. Большая часть этой работы была сделана во время моего визита в Математический институт Макса Планка (Бонн), и я хотел бы выразить свою признательность за гостеприимство и поддержку всем сотрудникам этого института. Я хочу поблагодарить Д. Ору, Д. Орлова, С. Белева и П. Бресслера за постоянную помощь, полезные обсуждения и замечания.

Работа выполнена при частичной финансовой поддержке РФФИ (гранты № 08-0100095 и 08-01-00392_а).

\section{Список литературы}

[1] M. Audin, Torus Actions on Symplectic Manifolds, Progr. Math., 93, Birkhäuser, Basel, 2004.

[2] Н. Нехорошев, Функи. анализ и его прил., 28:2 (1994), 128-129.

[3] S. A. Belyov, N. A. Tyurin, On non toric fibrations on lagrangian tori of toric Fano varieties, MPIM-2009-14, Max Planck Inst. Math., Bonn.

[4] T. Delzant, Bull. Soc. Math. France, 116:3 (1988), 315-339.

[5] N.A. Tyurin, "Geometric quantization and algebraic Lagrangian geometry", Surveys in Geometry and Number Theory: Reports on Contemporary Russian Mathematics, London Math. Soc. Lecture Note Ser., 338, ed. N. Young, Cambridge Univ. Press, Cambridge, 2007, 279-318.

[6] A. Ashtekar, T. Schilling, "Geometric formulation of quantum mechanics", On Einstein's Path, ed. A. Harvey, Springer, New York, 1999, 23-65.

[7] Н. А. Тюрин, ТМФ, 158:1 (2009), 3-22.

[8] D. Auroux, J. Gökova Geom. Topol., 1 (2007), 51-91.

[9] С. Белев, Нелинейные квантовые системы, Диплом магистра, ОИЯИ, Дубна, 2009.

[10] S. Belyov, Pseudo toric fibrations of non toric del Pezzo surfaces, in preparation.

[11] Н. А. Тюрин, ТМФ, 150:2 (2007), 325-337.

[12] N. Hitchin, "Lectures on Special Lagrangian Submanifolds", Winter School on Mirror Symmetry, Vector Bundles and Lagrangian Submanifolds, AMS/IP Stud. Adv. Math., 23, eds. C. Vafa, S.-T. Yau, AMS, Providence, RI, 2001, 151-182.

Поступила в редакцию 10.04.2009 